\title{
Chlorine dioxide (CLO2) as a non-toxic antimicrobial agent for virus, bacteria and yeast (candida albicans)
}

\begin{abstract}
The use of a family of chemical agents, releasing active species of oxygen, effective against infectious microorganisms and viruses is described with emphasis on Chlorine Dioxide $\left(\mathrm{CLO}_{2}\right)$, one of the oxides of chlorine. Also discussed are ozone, hypochlorite, periodate and the known mechanisms of action by which certain white blood cells attach and eradicate infectious microorganisms and primitive bacteria also known as viruses.

Explanation of the biochemical mechanisms of acid of $\mathrm{CLO}_{2}$ as an anti-microbial agent, is presented. Particular attention is given to Candida albicans, cytomegalovirus, polio virus, Herpes I and II, HTLV-III and Pseudomonas responding to the clinical application of CLO It is implied that these biochemical mechanisms are so fundamental that the development of resistant strains of bacteria and/or yeast would not occur with other anti-infectious agents. Limited lists of health abnormalities that respond to $\mathrm{CLO}_{2}$ are discussed.
\end{abstract}

Volume 2 Issue 6 - 2016

Robert OYoung

Universal Medical Imaging Group, USA

Correspondence: Robert O Young, pH Miracle Inc., 16390 Dia del Sol,Valley Center, California, 92082, USA, Tel 760 75। 832I, Email phmiracleliving@aol.com

Received: December 26, 2015 | Published: October 08, 2016

Keywords: chlorine dioxide, ozone, chlorite, chlorate, chloride.

\section{Introduction}

$\mathrm{CLO}_{2}$ is an inorganic compound composed of oxygen; chloride; potassium and gold. Compounds of oxygen and chloride are formed from two electronegative elements. This chemical property of $\mathrm{CLO}_{2}$ makes possible the release of nascent oxygen upon decomposition during its action as an anti-microbial agent. Certain aspects of the cellular immune system (specifically white blood cells) utilize other mechanisms in the generation of highly reactive oxygen derivatives (free radicals) for the purpose of combating the invasion of foreign organisms. Without these protective mechanisms provided by the immune system involving oxygen derivatives; the ability to fight infection is greatly impaired.

The immune system of many persons; particularly the elderly; is deficient in the ability of providing these highly reactive oxygen derivatives (free-radicals) which are so necessary for attacking the great variety of viral; and bacterial invaders become easy targets for the many diseases they produce with the accompanying and sometimes bizarre symptoms.

The use of $\mathrm{CLO}_{2}$ assists the natural protective mechanisms of the body in counteracting these infectious agents which; if not adequately neutralized; will most certainly lead to disease. There will be described below the biochemical mechanisms by which $\mathrm{CLO}_{2}$ assists the immune system in destroying pathogenic organisms by making available an active form of oxygen. ${ }^{1}$

\section{Clinical applications of anti-microbial agents \\ Physical properties and chemical structure}

$\mathrm{CLO}_{2}$ in pure form (anhydrous) is a liquid at $\mathrm{O}^{\circ} \mathrm{C}$. Having a deep red color. When mixed with water and at high dilution it is colourless. ${ }^{2}$

Bohr atomic models indicate a "coordinate covalent" bond between the elements of $\mathrm{CLO}_{2}$. This type of bond represents the sharing of a pair of electrons between two atoms as in the "covalent" bond; but in this bond both electrons are contributed by one of the atoms (chlorine) and none by the other. In the covalent bond one electron is contributed by each of the atoms forming the bond (Figure 1).

When $\mathrm{CLO}_{2}$ reacts as an anti-microbial agent the oxygen atom first binds to a single atom (the one being oxidized) and then is dissociated from chlorine. An electron is then given up to chlorine forming the chloride ion. When one realizes that there are $5.3 \mathrm{~g}$. of chloride ion per liter of human plasma it becomes obvious that the small amount of chloride generated through the use of $\mathrm{CLO}_{2}$ is negligible.

\section{Chemical definition of $\mathrm{CLO}_{2}$}

$\mathrm{CLO}_{2}$ is an inorganic (containing no carbon) sodium salt of only electronegative elements bound together by electrostatic; covalent and coordinate covalent bonds (Figure 2). The chemical structure of $\mathrm{CLO}_{2}$ is $\mathrm{pH}$-dependent; that is; the stability or instability of this substance is determined in large measure by the concentration of hydrogen ions $(\mathrm{H}+$ or protons $)$ in the surrounding medium.

Under acidic conditions; sodium chlorite becomes unstable and decomposes into a variety of products including but not limited to chloride ions; hyperchlorous ions and nascent (atomic) oxygen. It is for this reason that $\mathrm{CLO}_{2}$ when bottled and stored for long periods of time; is buffered. Under acidic conditions; $\mathrm{CLO}_{2}$ results in a neutral molecule consisting of three electronegative atoms held together by covalent and coordinate covalent bonds.

From this cluster; a single atom of highly reactive nascent oxygen is liberated onto the target microorganisms. As you will see below; nascent oxygen is the active agent of $\mathrm{CLO}_{2}$ and; unless it is liberated; the antiviral; antibacterial and antifungal activity will not occur.

\section{Virus inactivation}

A virus typically consists of an outer shell or coating of protein encapsulating a nucleic acid which may be either DNA or RNA (e.g. Retroviruses). The skeletal backbone of nucleic acids includes derivatives of phosphoric acid $\left(\mathrm{H}_{3} \mathrm{PO}_{4}\right.$; a very strong acid) in which two of the original three hydroxyl groups $(-\mathrm{OH})$ are substituted; leaving only one active hydroxyl group per phosphate. 


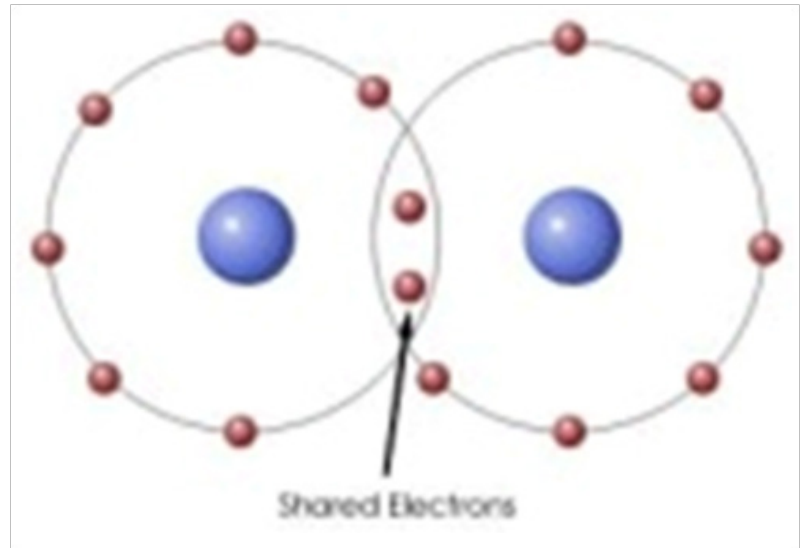

Figure I shows the sharing of electrons forming a covalent bond.

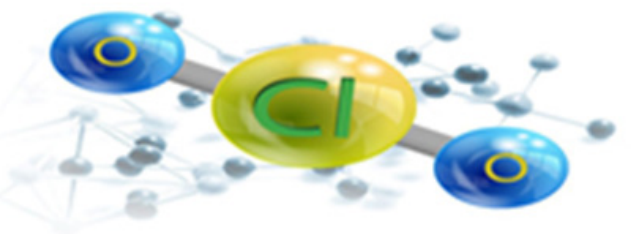

Figure 2 The Atomic Structure of $\mathrm{CLO}_{2}$.

\section{Segment of extended dna chain showing alternating deoxyribose- phosphate linkage}

Some viruses may have glycoproteins incorporated into their protein coat; that is; proteins to which polysaccharides (sugar chains) have been attached. The bound polysaccharides may attach to specific sites on the protein coat; effectively converting a protein surface into a poly- sugar surface. ${ }^{3}$ This new surface has specificity for certain polysaccharides found on the surfaces of specific cell types; thereby conveying specificity to virus binding as well as a degree of immunological protection.

Once bound to the appropriate cell type; the nucleic acid component of the virus is injected into the cell and in many ways; takes over the protein synthesis processes of the cell. Certain segments of the viral nucleic acid consist of genes that are responsible for the replication of the coat. The nucleic acid component replicates by a process known as "base-pairing"; in which each base of the original strand attracts and binds the corresponding base; forming a pair (A-T and C-G; described more fully below) through hydrogen bonding. The result is the replication of the complete virus until the cell bursts; releasing many additional viral particles into the surrounding medium. In the presence of these acidic nucleic acids; the $\mathrm{CLO}_{2}$ molecule becomes unstable and releases nascent oxygen into the medium.

Nucleic acids; both RNA and DNA; have many characteristics in common; in fact; they are almost chemically identical. Each consists of a chain of alternating sugar (a modification of ribose; deoxyribose) and phosphate groups; known as the "phosphate-sugar backbone". Attached to a specific carbon of each deoxyribose group is an organic ring compound known as a "base". Altogether; there are only four types of bases found in DNA; namely; guanine $(\mathrm{G})$; cytosine $(\mathrm{C})$; adenine $(\mathrm{A})$ and thymine $(\mathrm{T})$. It is the sequence of these four units along the chain that makes one segment of DNA differs from another. RNA differs chemically from DNA in that the base thymine is replaced by the base uracil (U); representing a very subtle biochemical difference. There is also a subtle difference in the sugar deoxyribose.

\section{The release of nascent oxygen from chlorine dioxide $\mathrm{CLO}_{2}$ in an acidic environment}

The base guanine; found in both RNA and DNA; is very sensitive to oxidation; forming 8-oxoguanine as the oxidation product. ${ }^{4}$ The release of $\mathrm{CLO}_{2}$ results in the oxidation of the guanine residue with the formation of 8-oxoguanine; thereby disallowing the replication of the viral nucleic acid by base pairing. Although the replication of the protein coat may continue; the formation of a complete functional virus has been blocked by $\mathrm{CLO}_{2}$ oxidation.

\section{Aerobic and anaerobic bacteria}

When life began on earth but before the presence of oxygen in the earth's atmosphere; bacteria consisted of what are known today as the anaerobes. These bacteria survive only in the absence of oxygen and include many pathogenic organisms. With the appearance of algae; carbon dioxide; present in large proportion in the primordial atmosphere; was converted by algae to carbohydrates; generating oxygen as a by product. At first; oxygen reacted with iron salts in the primitive oceans to generate extensive deposits of iron oxide (iron ore). When soluble iron in the oceans became exhausted; oxygen concentration in the atmosphere began to rise. At the present time; oxygen comprises approximately $20 \%$ of the earth's atmosphere while carbon dioxide levels have dropped to about $2 \%$.

Some bacteria were able to survive the increased levels of oxygen in the atmosphere by any of three paths. The presence of oxygen in the atmosphere resulting from algae and other plants containing the green pigment chlorophyll; allowed the development of the wide range of life forms both in the ocean and on land known today as animals. Some anaerobic bacteria took up residence in oxygen-free environments within the bodies of animals; notably; the intestines. Other anaerobes solved the problem of oxygen toxicity by evolving metabolic ways to cope with its universal presence. These include the development of the enzyme superoxide dismutase (SOD); which in its most primitive form; contained iron. ${ }^{5}$ Later refinements included the incorporation of manganese (in mitochondria) and finally; a combination of copper and zinc.

It is widely believed that still another solution to oxygen toxicity lay in the development of organisms that have the ability to utilize oxygen in their metabolism. These organisms are known today as the aerobes or aerobic bacteria. One form of these organisms may have been the precursor of an inclusion organism found in many animal and human cells known as the mitochondria; having its own circular DNA.

It is well known that cultures of many bacteria become acidic; typically generating lactic; acetic and other simple carboxylic (organic) acids. The acidic medium surrounding many bacteria triggers the decomposition of $\mathrm{CLO}_{2}$ and the subsequent liberation of nascent oxygen. Nascent oxygen is a particularly potent oxidizing agent for anaerobic organisms because it is essentially a free radical seeking not one; but two electrons. Anaerobic organisms have not developed adequate defences against the onslaught of oxygen; particularly nascent oxygen; and quickly succumb to its lethal action.

One possible mechanism for the liberation of nascent oxygen from the chlorite ion $\left(\mathrm{CLO}_{2}^{-}\right)$in an acidic environment involves the 
association or binding of $\mathrm{H}+$ (proton; normally bound to the oxygen of water as $\mathrm{H} 3 \mathrm{O}+$ ) to any of the three pairs of unused electrons in the outer shell of chlorine. This association is equivalent to the formation of hydrogen - chlorine $(\mathrm{H}-\mathrm{Cl})$ covalent bond. Because of the single electron deficiency of the chlorine atom; only one covalent bond is permitted at any one time. Since the $\mathrm{H}-\mathrm{Cl}$ bond is stronger (the $\mathrm{H}-\mathrm{O}$ bond of water is almost the strongest bond known) than the existing $\mathrm{O}-\mathrm{Cl}$ covalent bond; the $\mathrm{O}-\mathrm{Cl}$ bond is disrupted by the ejection of nascent oxygen. This leads to the dissociation of the acidic proton from chlorine and the formation of the hypochlorous ion (ClO-) and sodium hypochlorite $(\mathrm{NaOCl})$ and the release of nascent oxygen from chlorine dioxide in an acidic environment.

The decomposition of sodium chlorite to nascent oxygen and sodium hypochlorite in an acidic environment

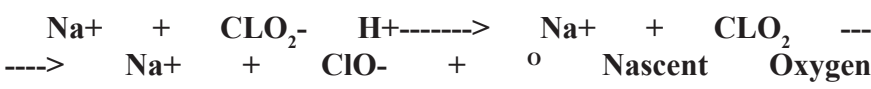
Sodium Ion + Chlorine Dioxide + Sodium Hypochlorous Ion + Ion Sodium Hypochlorite $>$ Nascent Oxygen

(Figure 3) The formula for decomposition of sodium chlorite to nascent oxygen and sodium hypochlorite in an acidic environment]. During World War I; this instability of chlorite ion was exploited by Alexis Carrel (1873-1944; Nobel laureate 1912); best known for his prolonged culture of chicken heart cells at Rockefeller University; New York. Carrel sucessfully administered a crude solution topically to war casualties having embedded shell fragments. ${ }^{6}$ The anaerobic organisms responsible for gas gangrene in these wounds were quickly quenched; saving many soldiers that would have otherwise perished.

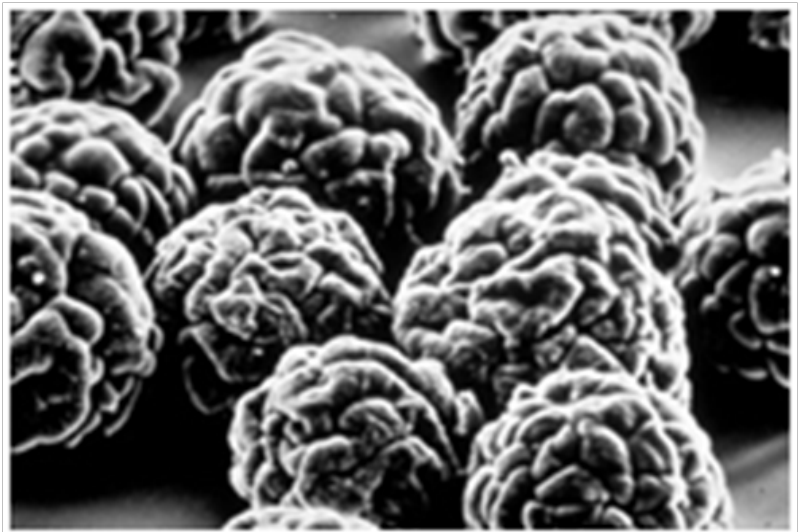

Figure 3 An electron micrograph showing the intact polio virus before exposure to ozone $\left(\mathrm{O}_{3}\right)$.

\section{Absence of $\mathrm{CLO}_{2}$ activation}

There are only a small number of basic biological substances found in living organisms. These include proteins; lipids; carbohydrates and nucleic acids. The ability of nucleic acids (in the form of viruses) to activate chlorine dioxide and then nascent oxygen has been discussed previously. Lipids and carbohydrates are both neutral substances; carrying neither an electric charge nor acidic groups. The possibility of proteins activating $\mathrm{CLO}_{2}$ is discussed below.

Many proteins; particularly those that are soluble and found free in both the blood and in the medium surrounding tissues; contain on their surfaces organic groups; both acidic and basic (alkaline) in nature. In most soluble (globular) proteins either one or the other of these two types of groups predominate. Proteins in which the acidic groups outweigh the basic groups are acidic proteins and those in which the basic groups predominate are known as basic proteins.

A technique has been developed to determine; for a given protein; in which category it may lie. Either acidic or basic proteins migrate under the influence of an applied electric field. This laboratory technique; known as electrophoresis; has been used to determine the acidity or basicity of proteins. As the $\mathrm{pH}$ of the surrounding medium into which a protein has been solubilized is changed; the protein will migrate either in one direction or the other. When a $\mathrm{pH}$ is found that will result in no migration; the protein is said to be at its isoelectric point. The $\mathrm{pH}$ value at which this occurs for a given protein is known as the isoelectric point of that protein. The isoelectric points of most proteins are not far from 7 or neutrality. Such proteins are not capable of activating $\mathrm{CLO}_{2}$ to decomposition; partly accounting for low toxicity.

\section{$\mathrm{CLO}_{2}$ as an antifungal agent}

Fungi are considered as plants without chlorophyll and lack the ability to generate carbohydrates from sunlight and carbon dioxide. They were probably derived in an oxygen-free atmosphere but some have developed the ability to tolerate low levels of oxygen. Most fungi prefer an oxygen-poor environment and live best under these conditions. They obtain their energy requirements from the decomposition (through enzyme activity) of existing organic matter and; in this light; may be considered as parasitic.

Because of their low tolerance for nascent oxygen and the acidic medium in which they thrive (from the liberation of organic acids); fungi in the mycelial form are sensitive to the destructive action of $\mathrm{CLO}_{2}$. One example of a human pathogenic fungus is Candida albicans; invading finger and toenails. A second example is the fungus responsible for "atheletes foot"; thriving between toes in a moist environment while still another example is "ringworm"; propagating on the dead cells found on skin (as well as generating many mycotoxins affecting homeostasis).

From all of the above considerations; it is apparent that $\mathrm{CLO}_{2}$ is essentially activated only by viruses; acidic bacteria and fungi. Other Cytotoxic Oxidizing Agents Used Clinically CL02 is not the only antimicrobial agent in clinical use. Another agent also providing active oxygen is hydrogen peroxide; which has been used in the treatment of arthritis; cancer and other metabolic diseases. Hydrogen peroxide is commercially available in low concentrations for the treatment of topical microbial infections.

Found to be more effective as a sporicide than as a bactericide; hydrogen peroxide is bacteriostatic at concentrations greater than 0.15 millimolar. ${ }^{8}$ Peracetic acid; $\mathrm{CHC}(0) \mathrm{OOH}$; is simply acetic acid; $\mathrm{CHC}(0) \mathrm{OH}$; with one additional oxygen atom inserted between the carbon and $\mathrm{OH}$ group. This bonding is unstable; decomposing to acetic acid and one atom of oxygen is being made available as bactericidal; fungicidal or virucidal. Peracetic acid exhibits rapid activity against spores and yeasts. ${ }^{8}$

An anti-microbial agent having chemical similarity to $\mathrm{CLO}_{2}$ is sodium hypochlorite $(\mathrm{NaOCI})$ in which only one atom of oxygen is bound to halogen rather than two. Solutions containing $125 \mathrm{ppm}$ were found effective within 30minutes in decontaminating objects which had been massively contaminated by both gram-positive and gramnegative bacteria and fungi. Drinking water heavily contaminated by a polymicrobial suspension became sterile in 30 minutes by a solution containing 10ppm of sodium hypochlorite. ${ }^{9}$

Still another oxidizing agent having increased application is ozone. 
Ozone is an unstable gas and is not commercially available but must be generated as required. It has been shown that ozone selectively inhibits the growth of human cancer cells. ${ }^{10}$ Under specified conditions cancer cell growth was inhibited more than 90 percent while control cells were inhibited less than 50 percent. ${ }^{11}$

These results indicate that cancerous cells; because of their altered oxygen metabolism; are less able to handle the oxidative stress presented by ozone than normal cells. Ozone has also been shown to inactivate polio virus with an effect noticeable after only 0.5 seconds of contact and is very strong after 2.5 seconds. These results parallel those caused by $\mathrm{CLO}_{2}$ in relation to polio virus (see below, antiviral activity of $\left(\mathrm{CLO}_{2}\right)$. Ozone is being used both in Europe and the U.S. to treat various diseases including a cancerous condition; blood coagulation disorders and liver diseases; among others ${ }^{12-14}$ (Figure 4).

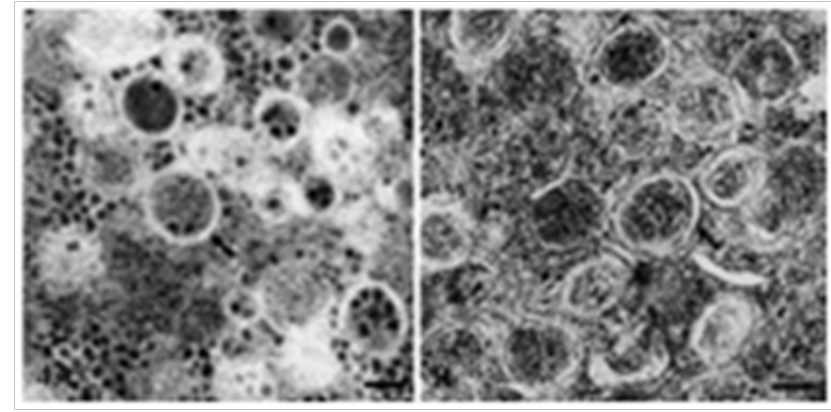

Figure 4 shows a Phase contrast micrograph of the polio virus after exposure to ozone $\left(\mathrm{O}_{3}\right)$.

\section{Cytotoxicity of $\mathrm{CLO}_{2}$}

Proof that $\mathrm{CLO}_{2}$ is cytotoxic to bacteria; fungus and virus clinically is shown by data indicating its effectiveness as a disinfectant (outside the body). $\mathrm{CLO}_{2}$ has been found to inactivate the organism causing Legionnaire's Disease (Legionella pneumophila). ${ }^{15}$ The chemically related compound sodium per iodate (NaIO4) inhibited the virulence; decreased the respiration of and increased the sensitivity to phagocytosis of the common pathogen Listeria monocytogenes. ${ }^{16}$

A germicidal solution has been developed containing $\mathrm{CLO}_{2}$ at an acid $\mathrm{PH}$ (lactic acid). The solution gave complete kill of Staphylococcus aureus; Pseudomonas and Candida albicans spores within 10minutes. If used in an ultrasound cleaning device complete killing occurred in less than five minutes. ${ }^{17}$ The bacterial virus $£ 2$ was rapidly inactivated with $\mathrm{CLO}_{2}$ at $\mathrm{pH} 5.9$ only GMP (guanosine monophosphate) reacted while the amino acids cystine; tryptophan and tyrosine reacted rapidly. ${ }^{18}$

$\mathrm{CLO}_{2}$ applied to polio virus separated the RNA from the protein coat (capsid). $\mathrm{CLO}_{2}$ reacted with the capsid protein and prevented the adsorption; penetration and normal un-coating of the virus. It also reacted with the viral RNA and impaired the ability of the nucleic acid to act as a template for replication. ${ }^{19}$

\section{Fate of $\mathrm{CLO}_{2}$ following ingestion}

The half-life for the elimination of $\mathrm{CLO}_{2}$ from experimental animals (rat) was 43.9 hours. When rats drinking $100 \mathrm{mg}$ of $\mathrm{CLO}_{2}$ per liter for 15 days were then given $3 \mathrm{ml}$ of $300 \mathrm{mg} /$ liter orally the half-life was 31 hours.

The distribution of $\mathrm{CLO}_{2}$ in tissues following administration of $\mathrm{CLO}_{2}$ labelled material is as follows

Plasma; 2.74; Kidney; 2.45; Lung; 2.25; Stomach; 2.15; Liver;
1.16; Spleen; 0.76; Thymus; $0.68 .{ }^{20}$ The metabolites of $\mathrm{CLO}_{2}$ are chlorite $\left(\mathrm{CLO}_{2}\right)$; chlorate $\left(\mathrm{CIO}_{3}\right)$; chloride $(\mathrm{CL})$ and oxygen $(\mathrm{O} 2)^{21,22}$ (Figure 5).

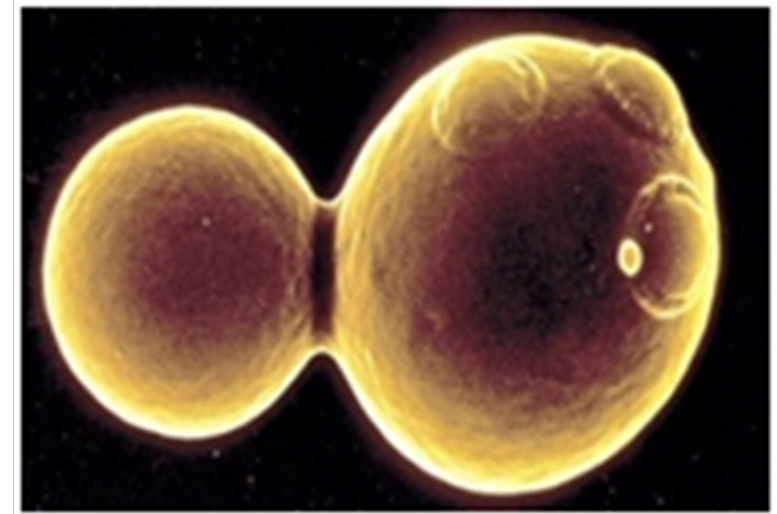

Figure $\mathbf{5}$ Is a scanning electron micrograph of Candida albicans showing the bud scars.

\section{Toxicity of $\mathrm{CLO}_{2}$}

It has been shown by toxicity studies in man that daily ingestion of $500 \mathrm{ml} \mathrm{CLO}_{2}$ having a concentration of $5 \mathrm{ppm}$ is safely tolerated. ${ }^{23} \mathrm{In}$ other studies with rats the LD of $\mathrm{NaCLO}_{2}$ was established as $140 \mathrm{mg} /$ $\mathrm{kg} .{ }^{2}$ In studies where $\mathrm{CLO}_{2} ; \mathrm{Na} \mathrm{CLO}_{2}$ or $\mathrm{CIO}_{3}$ was included in the drinking water for up to several months where no significant increases in methemoglobin concentrations resulted with doses as high as $1000 \mathrm{mg} /$ liter $(1000 \mathrm{ppm})$ in the rat; mouse or chicken. ${ }^{20}$

\section{$\mathrm{CLO}_{2}$ in wound healing}

It has been demonstrated clinically that $\mathrm{CLO}_{2}$ is highly effective in accelerating wound healing with particular applications to burns. There may at first be no apparent explanation for such a relationship but careful biochemical analysis has revealed a mechanism of action relating $\mathrm{CLO}_{2}$ to wound healing. Nucleic acids (RNA and DNA) are formed of only four basic units linked together into a chain. One of the basic units is designated; guanosine monophosphate; or GMP. If the phosphate group is brought close to one of the hydroxyl groups of the sugar component ribose; water is eliminated and the product is known as cyclic GMP (cGMP).

It has been shown that cGMP stimulates cell division and is activated during the regeneration process. It is also known that the enzyme synthesizing cGMP; guanylate cyclase; is stimulated by one of the highly reactive oxygen derivatives; hydroxyl radical; symbolized by $\mathrm{OH}-$. An oxidizing agent chemically similar to $\mathrm{CLO}_{2}$ is periodic acid; $\mathrm{HIO} 4$; in which four oxygen atoms are bound to a single halogen atom; iodine. In $\mathrm{CLO}_{2}$; two oxygen atoms are bound to the halogen chlorine. It has been determined that hydroxyl radical is present in solutions of periodic acid ${ }^{24}$ and; by inference; may also be present in solutions of $\mathrm{CLO}_{2}$. The presence of hydroxyl radicals in $\mathrm{CLO}_{2}$ activates the enzyme guanylate cyclase; which; in turn; synthesizes cGMP leading to cell proliferation and wound healing. ${ }^{1}$

\section{The significance of candida albicans in therapy with $\mathrm{CLO}_{2}$}

Candida albicans is a normal resident of the human digestive system; and vaginal tract and; unlike many other yeasts; is found in two forms (dimorphic). Baker's or brewers yeast is a familiar example of a yeast which occurs in only one form; that of single ovoid-shaped 
cells which reproduce by the process of "budding." An older; large cell constricts near one end and eventually closes off thereby forming a daughter cell which breaks free and continues the reproductive cycle.

Candida albicans is said to be "dimorphic" because the ovoid single-cell may; under certain conditions; continue to elongate into an almost perfect cylinder (mycelium); occasionally laying down a wall or partition separating individual cells. This process is also accompanied by branching in which a new tube may start to grow laterally from a specific point. By changing the culture conditions the form of Candida albicans may be changed from one form to the other and vice versa. This phenomena is known as pleomorphism (Figure $6)$.

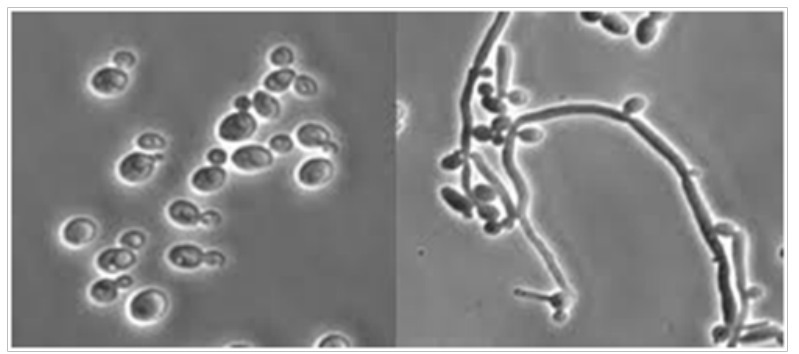

Figure 6 Y-form or round Candida albicans can biologically transform into a M-form or mycelial Candida albicans.

It is believed that only the mycelial form is pathogenic in man; who will be discussed in greater detail below. The incidence of both superficial and invasive candidosis has been increased markedly over the last few decades; probably resulting from the widespread usage of immunosuppressive treatment; antibiotics; etc. The organism can reasonably be described as the most common and most serious fungal pathogen of man..$^{25}$

Candida albicans has a worldwide distribution and is commonly found in normal individuals. The incidence of this fungus in various groups undergoing medical treatment is in general higher. Candida vaginitis is at present the second most common form of vaginal infection in the United States. Of the women who develop vaginal candidosis; a significant proportion suffer frequent recurrences or show chronic intractable symptoms. Candida albicans can infect virtually every tissue in the human body. Clearly there is a requirement for new and more effective antifungal drugs. ${ }^{26}$

For Candida albicans to successfully colonize and infect mucosal surfaces it has to adhere to epithelial surfaces. In germ free animals the mucosa is colonized in higher numbers than in that of control animals (normal). These results suggest that the indigenous bacterial flora suppresses Candida albicans colonization. This; in turn; implies that at least one factor contributing to Candida albicans infection may be the balance between the various components of the intestinal flora. ${ }^{26}$

\section{Lysis of candida by $\mathrm{CLO}_{2}$}

The lysis of Candida (and other infectious yeasts) by $\mathrm{CLO}_{2}$ may be accounted for by a simple mechanism related to the oxidizing ability of this substance. There follows a description and rationale for the mechanism as related to chitin; one of the constituents of the fungal cell wall. Chitin is a polymeric substance (formed by linking together a basic subunit) forming the bulk of the hard parts of insects (shell; legs; head; etc.). It is a water-insoluble substance formed by linking together the glucose derivative; $\mathrm{N}$-acetylglucosamine (NAG) (contains nitrogen while glucose does not). Chitin is biochemically synthesized by the enzyme chitin synthetase; and provides rigidity to the fungal cell wall. ${ }^{27}$

Yeast cells reproduce asexually by budding in which a daughter cell is formed by a constriction near one end of a mature cell. The construction continues until the walls almost meet followed by a thickening of the wall at this point. The space remaining is filled with a plug of chitin (ki-tin). When the daughter cell breaks free what would otherwise be an open hole in the cell becomes a bud scar of chitin plugging the hole and preventing cell disruption through lysis ${ }^{28}$ see Figure 3. Thus; any agent which inhibits the synthesis of chitin should; in the light of these considerations; act as a lytic agent for yeast.

In an effort to answer the question as to whether the cellular immune system (white blood cells) has an anti-fungal action against Coccidioides immitis; polymorphonuclear leukocytes (PMN cells) were incubated with the fungus under culture conditions. The results of contacting PMNs with the fungus indicated that the incorporation of NAG into chitin was inhibited from 54 to 85 percent. $^{28}$

PMNs from patients with chronic granulomatous disease (CGD) are apparently equivalent in every way to similar cells from normal individuals but lack the ability to generate active oxygen species (members of the Reactive Oxygen Toxic Species or ROTS family) used by these cells to fight infection (including superoxide; hydrogen peroxide; hydroxyl radical and singlet oxygen). One measure of the production of reactive oxygen toxic species or ROTS by these cells is the chemiluminescence (chemical generation of light) resulting from the presence of foreign particles. Tests made with PMNs isolated from patients with CGD are remarkably low in chemiluminescent response to stimulation.

When PMN's from a patient with CGD were incubated with Coccidioides; the incorporation of NAG into chitin was inhibited from only 0 to 22 percent under various conditions as compared to 54-85 percent with normal PMNs. ${ }^{28}$ These results indicate that the agents responsible for the inhibition were the highly reactive oxygen toxic species (ROTS) mentioned above. Similar oxidative species arise from the breakdown of certain oxidizing agents (including $\mathrm{CLO}_{2}$ ) when in contact with the target substances (bacterial proteins; polysaccharides; viruses; etc.). It has been shown that periodic acid (HIO4); a substance in which four oxygen atoms are bound to iodine (corresponding to chlorine in $\mathrm{CLO}_{2}$ ); is capable of forming one of the ROTS family; hydroxyl radical. ${ }^{24}$

Another reactive oxygen derivative; superoxide; may result from the breakdown of $\mathrm{CLO}_{2}$ (during an oxidative reaction) in which the two oxygen atoms released acquire an electron. It is known to be produced by Candida albicans as a metabolic by-product. ${ }^{29}$ Thus; three members of the ROTS family of oxygen derivatives; namely; hydroxyl radical; superoxide and singlet oxygen; are produced by both PMNs during the oxidative burst (response to foreign particulate matter) and $\mathrm{CLO}_{2}$ during an oxidative process. These considerations indicate that $\mathrm{CLO}_{2}$ as well as PMN cells are capable of inhibiting the biosynthesis of chitin in Candida resulting in lysis from the absence of the chitin plug at the bud scar.

\section{Antiviral activity of $\mathrm{CLO}_{2}$}

Viruses; in general; consist of two parts; an inner nucleic acid core consisting of either; DNA or RNA; and an outer protein coat. The protein coat not only serves to protect the vital nucleic acid within but is also instrumental in providing specificity in binding to the surface of particular cells (polio virus binds to nerve cells; influenza virus binds 
to the nasal mucosa; etc.). It has also been shown that without certain specific polysaccharides attached to the protein coat (glycoproteins); binding to the cell surface will not occur. ${ }^{30}$

\section{This aspect will be discussed in greater detail below in relation to the action of $\mathrm{CLO}_{2}$ on viruses}

Some virus; including the AIDS virus HTLV-I3I; herpes and cytomegalovirus; are surrounded by a bilayer lipid membrane which is a fragment of the plasma membrane of the cell in which the virus replicated. As the virus ruptures and kills the cell it takes with it a fragment of the plasma membrane of that cell and becomes encapsulated. This membrane is critical for infectivity and without it the virus cannot enter the proper cell type for replication. Anti-viral agents which damage or deteriorate the encapsulating bilayer lipid membrane also inhibit or prevent infectivity. We shall return to this aspect of the anti-viral activity of $\mathrm{CLO}_{2}$ in a subsequent section.

\section{Biochemical activity of $\mathrm{CLO}_{2}$}

The action of $\mathrm{CLO}_{2}$ as a cytotoxic agent may include as many as four separate biochemical mechanisms described below.

\section{Oxidation of protein}

As mentioned above; the protein coat is highly significant in viral infectivity and provides specificity for binding to the proper cell type. Two amino acids that would undergo oxidation and modification to a significant enough degree to permit inhibition of virus to cell binding are those containing sulfur; namely; methionine and cysteine.

The residue of methionine; $-\mathrm{CH}^{\wedge} \mathrm{CH}^{\wedge} \mathrm{CRj}$; contains the $\mathrm{C}-\mathrm{S}-\mathrm{C}$ thioether linkage subject to oxidation in either one or two stages. In the first stage the binding of one atom of oxygen results in the formation of the sulfoxide - $\mathrm{CH} 2 \mathrm{CH} 3 \mathrm{SCO} 2 \mathrm{CH}$; having the ability to bind several molecules of water (hydrophilic). The parent compound; methionine; is hydrophobic (in relation to the residue). A subsequent stage of oxidation leads to the formation of the sulfone; $-\mathrm{CH}_{2} \mathrm{CH}_{2} \mathrm{~S}\left(\mathrm{O}_{2}\right) \mathrm{CH}$; which is even more hydrohpilic than the sulfoxide. Both methionine sulfoxide and sulfone are well known modifications of this amino acid. $^{29}$

The modification of the water binding ability of a protein constituent (methionine) is highly significant in relation to the antiviral activity of $\mathrm{CLO}_{2}$. In transmission electron micrographs of virus which has been exposed to the action of $\mathrm{CLO}_{2}$ it appears that the outer coat has swollen much like a sponge. The increased penetration of water into the viral protein coat allows additional $\mathrm{CLO}_{2}$ (water soluble) to penetrate to still deeper layers of the coat; thus initiating a vicious cycle (Figure 3 ).

\section{Oxidation of nucleic acid (RNA)}

As mentioned above; $\mathrm{CLO}_{2}$ is capable of generating a variety of extremely reactive oxygen derivatives when acting as an oxidizing agent. Another example of an oxidizing agent releasing atomic or nascent oxygen is ozone; $\mathrm{O}_{3}$ this molecule consists of a chain of three oxygen atoms held together by covalent bonds. The release of one atom of oxygen results in stable; molecular oxygen; 0 . The oxidizing activity of $\mathrm{CLO}_{2}$ is similar to that of ozone; liberating highly active forms of oxygen. Very detailed research has been described related to the oxidation of polio virus by ozone. Exposure of the virus RNA strand to ozone resulted in fragmentation into several short chains. The effect of ozone on the nucleic acid was measured spectrophotometrically indicating that inactivation was complete after only sixty seconds. ${ }^{30}$
Nucleic acid comprising the RNA or DNA of viruses consists of a chain of four building blocks arranged in some specific sequence. It is the sequence of the four nucleotides (designated by the letters A; $\mathrm{C} ; \mathrm{G}$ and $\mathrm{U}$ for RNA) that causes one strand to differ from another resulting in the synthesis of specific proteins for each. When a mixture of the four nucleotides is exposed to ozone it is found that only the unit designated; $\mathrm{G}$ (guanine); is degraded in the initial stage. In the exposure of transfer RNA (tRNA) to ozone; guanine again was the first nucleotide to be degraded. Exposure of tobacco mosaic virus (TMV) to ozone caused a rapid loss of infectivity within 30minutes showing a preferential degradation of guanine. ${ }^{2}$

\section{Oxidation of lipids}

Some viruses are surrounded by a bilayer lipid membrane as described above. Any substance which acts to disrupt this membrane is an anti-viral agent for that virus since the membrane is essential for infectivity. $\mathrm{CLO}_{2}$ may act to disrupt this membrane by peroxidizing the unsaturated fatty acids found in the bilayer lipid membrane. The specific positioning of these unsaturations along the fatty acid chain is related to the integrity and fluidity of the membrane. The peroxidation of unsaturations results in the development ofhydrophilic (water seeking) groups in the central part of the fatty acid chain which causes distortion in the bilayer lipid membrane. This activity of $\mathrm{CLO}_{2}$ has not been demonstrated biochemically but is very apparent in the electron micrographs (transmission) of viral membrane disruption shown in Figure 10.

\section{Oxidation of polysaccharides}

There are found on the surfaces of viruses; bacteria and fungi (including C. albicans) chains of sugars (polysaccharides); some attached to protein as glycoprotein. An example of such a polysaccharide is mannan composed principally of sugar mannose and found on the surface of many yeasts. The degradation of sugars and polysaccharides has been conducted mainly with periodic acid and its salts having the structure $\mathrm{HIO}_{4}$. The oxidizing part of this substance is the ion $\mathrm{IO}_{4}$ - (periodate); in which four oxygen atoms are bound to a single atom of iodine (halogen). A similar oxidizing agent is $\mathrm{pH}$ Miracle's $\mathrm{CLO}_{2}$ product called Activator having the structured $\mathrm{CLO}_{2}$ or in its partially reduced form; CLO.

When an oxidizing agent of this nature is applied to the free aldehyde group of a "reducing sugar" (one in which the ring has opened with the formation of an aldehyde) an extensive degradation is initiated as exemplified by the equations ${ }^{31}$ (Figure 7).

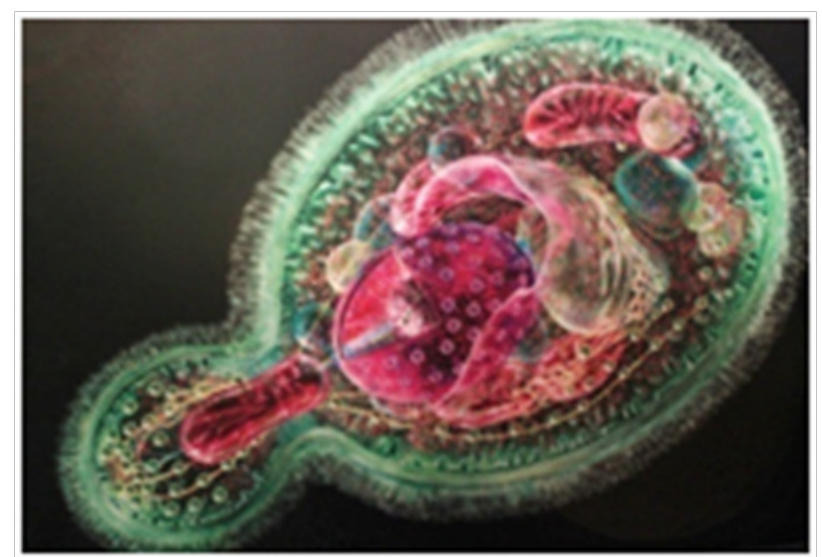

Figure 7 Electron micrograph of Yeast Cell Wall. 


\section{H-C-OH illustration}

The products formed are low molecular weight substances including formaldehyde; formic acid; carbon dioxide; etc. The surfaces of many organisms and viruses carry polysaccharides that are highly significant in relation to infectivity. It is these polysaccharides showing specificity for the surface of target cells that enables the organism to bind to those particular cells and invade (infectivity). ${ }^{31}$ One of the mechanisms of action of $\mathrm{CLO}_{2}$ against pathogenic organisms (including Candida) is undoubtedly the oxidation and degradation of sugars with a concomitant loss of infectivity and integrity for those organisms.

\section{Significance of the cell wall of candida albicans}

The principal cell wall polysaccharides of most yeasts are mannans (formed mainly of the sugar; mannose) and glucans (formed mainly of the sugar; glucose) whose basic structures have been determined. In models of the yeast cell wall recently proposed; a mannan-protein component is found in the outer region while glucan is found mainly in the inner region. Chitin (a polymer of the glucose derivative; $\mathrm{N}$-acetylglucosamine) has been detected not only in the bud scar but also in the glucan network of the cell wall. ${ }^{32}$

Treatment of the cells (yeast form) of C. albicans with helicase; an enzyme preparation from snail gut; releases mostly mannose from the surface during the first 20minutes. Holes are formed in the cell wall which allow further penetration into the inner glucan layer during the next 40minutes (Figure $5 \& 8$ ). Using selective stains for electron microscopy as many as eight discrete layers have been demonstrated in the cell wall of C. Albicans. The outermost layer; 1 ; consists mostly of 1;6-mannan. The second layer is composed of 1;2-mannan while the third layer is 1;6-glucan. The fourth layer is largely 1;3-glucan with the fifth layer; chitin. The innermost layers; 6;7 and 8; are composed largely of chitin-protein. These results confirm those described previously employing chemical extraction and enzymic digestion of the individual layers ${ }^{33}$ (Figure 8)

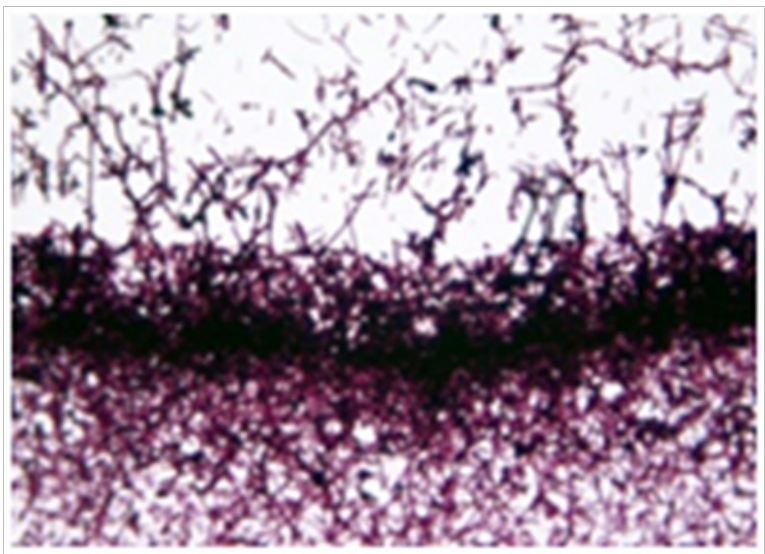

Figure 8 Shows the cell wall following digestion by the enzyme preparation, helicase.

Candida albicans is known as a "dimorphic" yeast; implying that it may exhibit either of two forms. One form; the yeast or Y-form (Figure 9A); is characterized by individual; ovoid-shaped cells similar in appearance to baker's yeast The second form is the mycelial (Figure 9B \& C); filamentous of $\mathrm{M}$-form; characterized by long; tubular threads separated at irregular intervals by a wall (septum). The Y- form reproduces by budding in which an older cell constricts at a point near one end and continues to narrow until a new daughter cell is pinched off. The M-form continues to elongate at the top; laying down a septum as it progresses. This form also branches at irregular intervals with the formation of a new mycelium ${ }^{34}$ (Figure $9 \& 10$ ).

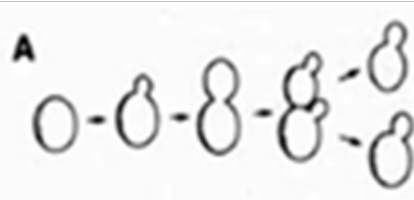

B
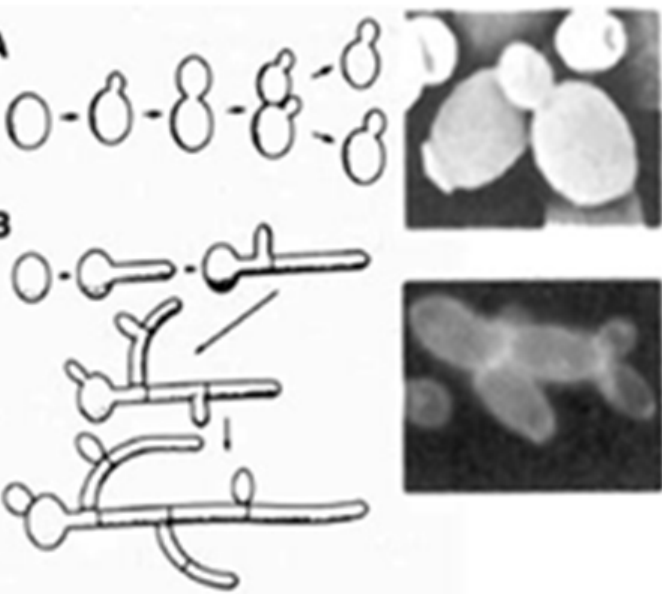

C

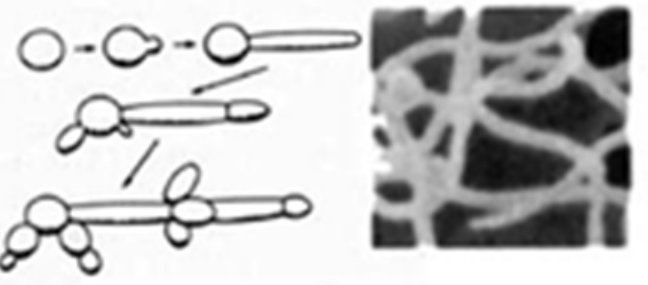

Figure 9 Electron Micrograph of Morphology of Candida albicans.

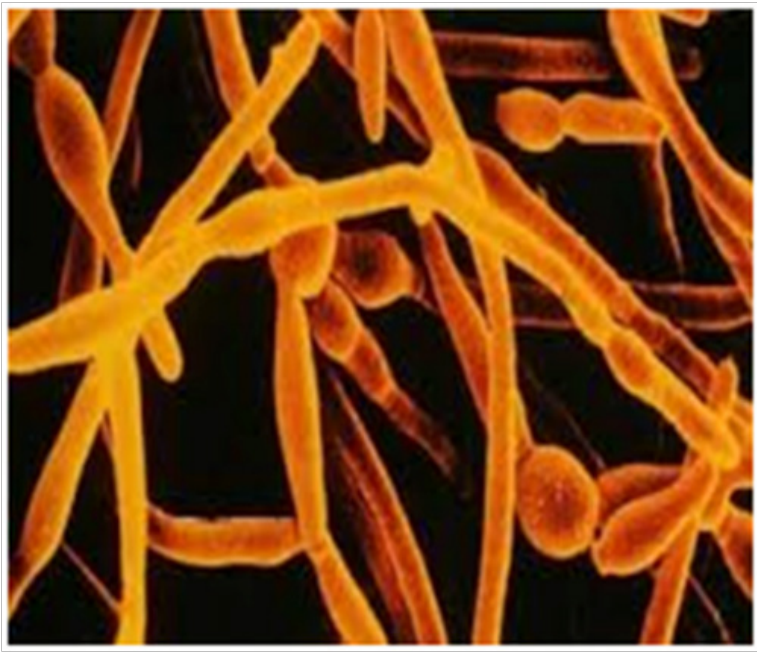

Figure I0 Electron Micrograph of a Mycelial Form of Candida albicans.

A great effort has been made by researchers to determine what factors or set or culture conditions are responsible for the $\mathrm{Y}$ to $\mathrm{M}$ conversion in C. albicans and vice versa. No one single causative factor has yet been defined although there is evidence that the factors are complex. Much attention has been given to this subject because there is a large body of literature that claims a relationship between the mycelial (M-form) and infectivity. ${ }^{25-36}$ This fungus is usually found in the Y-form in normal healthy individuals. ${ }^{36}$ The association of the M-form with pathogenicity is based on the premise that hyphae (filaments) penetrate tissues more readily than yeast cells and are more difficult to phagocytose. ${ }^{35}$

Figure 10 illustrated differences in morphology in spore development at two different temperatures; $20^{\circ}$ and $37^{\circ} \mathrm{C}$. In growing 
C. albicans in a chemically defined medium it has been found that biotin is essential for supporting maximal growth of various strains of this fungus. ${ }^{36}$ In cultures; supplementation with 1.0 millimicrogram/ $\mathrm{ml}$ of biotin. The biotin level of normal human blood as well as several tissues such as liver and brain is known to be in the range of 0.2-1.0 -' millimicrogram $/ \mathrm{ml}$. For these reasons there is a possibility that this vitamin plays a very definite role in determining the extent and the phase of Candida albicans growth in vivo. ${ }^{36}$

When the polysaccharide composition of cell walls isolated from normal-biotin and biotin-deficient cultures was analyzed it was found that the mannan (outer layer) content of biotin-insufficient cultures was 20 percent less than that of biotin-optimal cultures while the glucan (inner layer) content was 50 percent or more greater in biotindeficient cultures. ${ }^{36}$ It has also been found that almost all species of yeast contain mannan but filamentous fungi do not. ${ }^{36}$

In cultures of both baker's yeast and C. Albicans it has been pointed out the association of biotin and lipid; particularly those containing oleic acid; with the cell wall glucomannan- protein complexes and the involvement of biotin in the synthesis of lipid associated with the cell wall. It is possible that various alterations of cell wall lipids based on impaired fatty acid metabolism (catalyzed by biotin) lead to the overproduction of glucan at the expense of mannan. ${ }^{36}$

It has been shown that the Y-form of Candida albicans is favored over the M-form by normal amounts of biotin and that biotin deficiency leads to increased $\mathrm{M}$-form growth. This observation is highly significant in relation to the above mentioned association of the M-form with infectivity and pathogenicity. The implications are that treatment of Candida infections with $\mathrm{CLO}_{2}$ could be assisted by concurrent supplementation with biotin.

Yeast cells (Y-form) of C. albicans produce a heat-stable; low molecular weight substance that suppresses the yeast to mycelial transition with the inhibitory effect being increased by cobalt. ${ }^{28}$ Candida albicans germination in liquid medium was inhibited by sodium butyrate..$^{37}$

These combined research findings suggest that those who have adequate biotin; cobalt and sodium butyrate in their diet are less prone to develop the symptoms of yeast infection like Candida infection than those who are lacking these dietary supplements.

Some forms of bacteria appear as "pleomorphic" organisms or cell wall-deficient modifications of the normal structure. These modifications are also known as "L-forms" or dormant states in honor of the Lister Institute where they were classified as bacteria.

The active participation of L-forms in acute and chronic infection is just beginning to receive recognition. Candida also has been shown to possess a L-form having been isolated from the blood of patients treated with antibiotics such a penicillin or its derivatives. The mechanism of action of the penicillin is to interfere with the formation of a normal cell wall. Antibiotics of this nature which inhibit the formation of a complete cell wall are ineffective against the L-forms because they survive in the absence of a complete cell wall. ${ }^{38}$

\section{The Mechanism of action of several anti-fungal agents}

One class of drugs commonly used to treat Candida and other fungal infections is the so- called "polyene antibiotics" including nystatin; amphotericin B; filipin and others. These compounds are so-named because they consist of large organic rings in which half of the molecule is composed of a chain of unsaturated hydrocarbon (carbon-carbon double bonds alternating with single bonds) with the other half carrying many oxygen atoms; mostly as hydroxy groups $(-\mathrm{OH})$. In organic chemistry the term "ene" refers to a carbon-carbon double bond or "unsaturation;" hence the name polyene antibiotic. ${ }^{39}$

Research with these antibiotics has shown that one of the first observable effects after addition to a culture of Candida albicans is the release of intracellular potassium $(\mathrm{K}+)$. It has been assumed that cessation of growth results from the loss of potassium and other low molecular weight constituents of the cell and the simultaneous uptake of protons $(\mathrm{H}+)$ to balance the cell electrically. Support for this hypothesis comes from the finding that the growth inhibitory effects of these substances are prevented by the addition of potassium to the culture medium. However; other experiments may indicate that there are other mechanisms operating in addition to the loss of potassium through pores in the cell wall. ${ }^{40}$

From an inspection of the molecular structure of the polyene antibiotics it would appear that the molecule; when stacked upon it; forms the pore through which the ions pass. This; however; has been shown through a study of models of these substances; not to be the case. A model of amphotericin B indicates that each half of the circle is a rigid rod and that the two halves lie parallel to each other with no space between them for an ion to pass. ${ }^{41}$ This paradox is resolved by the realization that the pore or ion channel is formed from eight amphotericin B molecules arranged in a circle perpendicular to the membrane; forming octagonal channel. Two such pores are required to span the bilayer lipid membrane. The side of the molecule carrying the hydroxy groups lie on the inside of the pore; binding water and forming a hydrophilic channel through which ions and low molecular weight water-soluble substances may pass. The outside of the pore consists of unsaturated hydrocarbons (polyene) bound to sterols normally found in the cell wall (for example; ergosterol).

It has been shown that the complex with sterols is a necessary requirement for the fungicidal activity of these compounds resulting in a reorientation of sterols within the membrane structures..$^{42}$ What has been said in relation to amphotericin B also applies to nystatin. ${ }^{43}$

These aspects of molecular biology are in keeping with scanning electron micrographs (SEM) made of both growing and nongrowing cells of C. albicans. Nystatin influenced the function of the cell membrane in such a way as to induce distortions and deformations in the cell surface. ${ }^{44}$

\section{The alternate pathway in candida albicans respiration}

The fundamental equation of Candida albicans respiration is given by: $\mathrm{O} 2+4 \mathrm{H}++4 \mathrm{e}---$

Representing the reduction of molecular oxygen to water in which $\mathrm{H}+$ represents a hydrogen ion (proton) and e- represents an electron. One of the membrane-bound enzymes in the respiratory chain is cytochrome which binds molecular oxygen to a single atom of iron held tightly in an organic ring.

It is well known that cyanide; or more correctly cyanide ion $(\mathrm{CN})$; is highly toxic to organisms utilizing oxygen. This is partly so because cyanide ion binds more tightly to the iron atom in cytochrome than oxygen thereby displacing and preventing it from binding. When oxygen cannot bind to the iron the normal respiration process is inoperative.

Candida albicans; for optimum growth; requires oxygen and; in the complete absence of oxygen; growth almost ceases. ${ }^{25}$ This fungus possesses a cytochrome respiratory system which would be expected 
to be inhibited by cyanide ion. In the presence of cyanide respiration is not inhibited but is actually stimulated. This apparent stimulation of oxygen uptake by cyanide can most probably be explained on the basis of its inhibitory effect on catalase activity. Catalase; the enzyme which destroys hydrogen peroxide; is present in C. albicans 24 and contains an organic iron containing ring similar to that in cytochrome and hemoglobin. The degradation of hydrogen peroxide generates oxygen required for optimum growth.

The inability of cyanide to inhibit respiration has led to the proposal of an "alternate pathway" in Candida not involving cytochrome. It has been estimated that in growing cells the alternate pathway contributes less than 10 percent of the total respiration ${ }^{25}$. It is present in all strains of C. albicans thus far tested and is found in both the yeast and mycelial forms. Clear evidence indicates that the alternate pathway is located in the mitochondria. The exact biochemical mechanism for this respiratory pathway is at present unknown.

The presence of an alternate respiratory pathway may be highly significant in relation to one mechanism of $\mathrm{CLO}_{2}$ anti-fungal activity. The blockage of the alternate pathway by one of the highly reactive oxygen derivatives; for example; superoxide or hydroxyl radical; would eliminate the only remaining way in which the organism could survive.

\section{Sulfur metabolism in candida albicans}

Organic disulfides have the linkage; C-S-S-C; and are formed from two cysteine residues according to the equation: C-SH + HS-C + O----_------ > C-S-S-C + I^

When hydrogenated (reduced); the reaction is
reversible yielding the original cysteine residues as: C-S-S-C + $2 \mathrm{H}--->\mathrm{C}-\mathrm{SH}+\mathrm{HS}-\mathrm{C}$

This reversible reaction represents a form of cross-linking among protein strands which are; in fact; simply polymers of amino acids (linked together in chains). The result of cross-linking in protein; either within the same strand or between different strands in close proximity; is the formation of a more rigid structure since the motion of the strands is inhibited through cross- linking.

This principle has been successfully applied commercially in the "Permanent wave" treatment for hair in which the existing disulfide bonds are broken by one solution and reformed by another solution. While the second solution is reforming the cross-links; the hair is placed in the desired position which becomes fixed in that position when the solution is removed.

In normal cells of $\mathrm{C}$. albicans there is found an enzyme; protein disulfide reductase; which provides hydrogen to the disulfide linkage (rigid form) converting it to the sulfhydryl form (free motion). In a C. albicans mutant (an abnormal variation produced by radiation; etc.) This enzyme is absent or nonfunctional and results in cells that cannot divide (divisionless). These observations indicate that the cell division process is related to the ability to break disulfide bonds thereby converting the rigid protein to a more free or flexible form may be more desirable. These results imply that if disulfide bonds present in growing cells cannot be broken with the formation of sulfhydryl groups (-SH); cell division may not occur.

An agent preventing the formation of the sulfhydryl groups critical for cell division is $\mathrm{CO} 2$. In the equation shown above; one atom of oxygen is required for every two sulfhydryl groups in the removal of hydrogen and the formation of water. If these sulfur-containing groups are continually kept in an oxidized state (as C-S-S-C); cell division cannot occur and replication is prevented. This may be one of the mechanisms of action of $\mathrm{CLO}_{2}$ whereby the growth of Candida is inhibited. ${ }^{29-49}$

\section{Clinical application of $\mathrm{CLO}_{2}$}

Ongoing research at the Innerlight Biological Research Foundation has been investigating the clinical usage of $\mathrm{CLO}_{2}$ for the following applications for over twenty-five years.
a. Herpes I and HI
b. AIDS (HTLV-m) $)^{45}$
c. Sty (eye)
d. Cold sores (herpes)
e. Shingles (herpes)
f. Warts
g. Cytomegalovirus
h. Polio
i. Influenza
j. Canker sore
k. Ear infection
1. Chronic bronchitis Sinusitis
m. Dandruff
n. Psoriasis
o. Strep throat
p. Pseudomonas Pneumonia
q. Peridontal disease
r. Bladder infections
s. Mycotic (Fungal)
t. Candida (systemic)
u. Candida (snails) $)^{45}$
v. Candida (vaginitis)
w. Candida (oral; thrush)
x. Candida (gastrointestinal)
y. Ringworm Scleroderma

This research also includes nonspecific spores; bacteria and virus.

\section{Clinical outcome studies of $\mathrm{CLO}_{2}$}

Extensive clinical applications of $\mathrm{CLO}_{2}$ to Epstein-Barr virus (EBV); ${ }^{46}$ cytomegalovirus (CMV); hepatitis virus A; B; HIV (AIDS virus) and others are being used continually. The DNA of EBV within the virus itself is in a linear form. Sometime after infection; the ends are linked together; forming the circular form (episome). Once this form of DNA is firmly established; the cell is said to be in a latent state. The virus remains in this state in certain B-cells for the remainder of the patient's life. About $10 \%$ of the B-cells are in the actively proliferating state. ${ }^{47}$ An Epstein-Barr clinical study was conducted in the American Biologics Medical Center over a four year period from 1992 to $1996 .{ }^{46}$ 
Case load:There were 1207 patients treated with the Dioxychlor ${ }^{\circledR}$ protocol. 784 patients were female - 65\%. 423 patients were male $35 \%$. Ages ranged from 16 to 52 years.

Initial status: High IgG serum titers ranged from 400 to 5800 . Intensive treatment for 14days. Nutritional Supplementation. Sodium Chlorite $\mathrm{CLO}_{2}$.

\section{Therapy (baseline):}

a. Sodium chlorite $\mathrm{CLO}_{2}$ - intravenous drip; $10 \mathrm{cc}$ in $100 \mathrm{cc}$ saline; daily Intravenous studies in the 90's at the American Biologics Medical Center have established that $10 \mathrm{ml}$ of 25;000 ppm of Sodium Chlorite $\mathrm{CLO}_{2}$ in $100 \mathrm{ml}$ of physiologic saline administered over 30 minutes is a safe dosage level.

b. Sodium chlorite $\mathrm{CLO}_{2}$ - sublingual; 10 drops under tongue; twice daily.

c. Thymus extract (im) - weekly.

d. Vitamin C -15 g parenteral (drip).

Results (averages):Minimun time clinical improvement: 3days significant clinical improvement: 10 - 20days Antibody (IgG); $90 \%$ reduction; $<35$ days. ${ }^{48}$

\section{Microbiological Laboratory Studies:}

a. In 1986; the Microbiology Laboratory at Stanford University performed a series of tests showing the efficacy of $\mathrm{CLO}_{2}$ in neutralizing a variety of viruses. The concentration of $\mathrm{CLO}_{2}$ used was $0.75 \mathrm{ppm}$ in all the studies.

b. The viruses included Herpes II; HTLV III and Cytomelagalovirus. The study also included the bacterium Pseudomonas. Electron micrographs show the complete eradication of the viruses and Pseudomonas following treatment. ${ }^{49}$

\section{Acknowledgments}

None.

\section{Conflicts of interest}

Author declares there are no conflicts of interest.

\section{Funding}

None.

\section{References}

1. Robert YO. Mycotoxicology: The Study of Fungu Produced Mycotoxic Species (FPMS) and Their Metabolism in Health and Disease. The Innerlight Biological Research Foundation, Alpine, USA. 1985.

2. Kirk-Othmer Encyclopedia of Chemical Technology. (3rd edn), McGraw-Hill Book Co, New York, USA. 2014. p.612.

3. Brack AR, Klupp BG, Granzow H Rebecca T, et al. Role of the cytoplasmic tail of pseudorabies virus glycoprotein $\mathrm{E}$ in virion formation. Journal of Virology . 2000;74(9):4004-4016.

4. de Souza-Pinto NC, Eide L, Hogue BA, et al. Repair of 8-oxodeoxyguanosine lesions in mitochondrial DNA depends on the oxoguanine DNA glycosylase (OGG1) gene and 8- oxoguanine accumulates in the mitochondrial DNA of OGG1-defective mice. Cancer Res. 2001;61(14):5378-5381.

5. Choi DH, Na BK, Seo MS, et L. Purification and characterization of iron superoxide dismutase and copper-zinc superoxide dismutase from Acanthamoeba castellanii. J Parasitol. 2000; 86(5):899-907.
6. Carrel A. The Book: Man. 1935.

7. Carrel A, Dehelly G. The treatment of infected wounds. (2nd sedn), Tindall \& Cox, London, UK. 1918. p.238.

8. Baldry MGC. The Bactericidal, Fungicidal and Sporicidal Properties of Hydrogen Peroxide and Peracetic Acid. Journal of Applied Microbiology. 1983;54(3):417-423.

9. Mastroeni P, Stassi G, Focà A, ET AL. Kinetics of the Antibacterial and Antimycotic Activity of an Anteseptic Solution on Different Substrates. Chem. Abssts. 1983;34(1):63-78.

10. Sweet F, Kao MS, Lee SC, et al. Ozone Selectivity Inhibits the Growth of Human Cancer Cells. Science. 1983;209(4459):931-933.

11. Pichtman EG. Chemical and Viricidal Investigation of the Ozonization of Waste water Systems. Proceedings - Forum on Ozone Disinfection. 1976.

12. Wolff H. Normal Cases of Ozone Therapy. Erfahrungsheilkinnde, Germany. 1975.

13. Viebahn R. Physicochemical Basis off Ozone Therapy. Erfahrungsheilkinnde, Germany. 1975.

14. Kief H. New Possibilities in Ozone Therapy. Erfahrungsheilkinnde, Germany. 1980.

15. Berg JD, John CH, Paul VR, et al. Growth of Legionella pneumojphila in Continuous Culture. Applied and Environmental Microbiology. 1985;49(6):1534-1537.

16. Velyanov D. Biological Changes in Listeria Monocytogenes After Treatment with Some Chemical Substances. Chem Absts. 1979;21:14514.

17. Alliger H. Germicidal Composition. Chem Absts. 1980.

18. Noss CI, et al. Reactivity of Chlorine Dioxide with Nucleic Acids and Proteins. Chem Absts.

19. Alvarez ME, O'Brien RT. Mechanisms of Inactivation of Poliovirus by Chlorine Dioxide and Iodine. Appl Environ Microbiol. 1982;44(5):1064-1071.

20. Abdel-Rahman MS, Couri D, Bull RJ. Kinetics of Chlorine Dixoide and Effects of Chlorine Drinking Water on Blood Glutathione and Hemolysis in Rat and Chicken. J Environ Pathol Toxicol. 1979;3(1-2):431-449.

21. Magda M, Francesc G. Stability of Chlorine Dixoide in Aqueous Solution. Water Research. 1982;16(9):1379-1382.

22. Abdel-Rahman MS, Couri D, Jones JD. Chlorine Dixoide Metabolism in Rat. J Environ Pathol Toxicol. 1979;3(1-2):421-430.

23. Bianchine JR, Judith RL, Sudha C, et al. Study of Chlorine Dioxide and Its Metabolites in Man National Service Center for Environmental Publications. 1982.

24. Scott JE, Page DPT. Spectrofluorometric Detection and Measurement of Hydroxyl Radicals in Periodate Solutions. Carbohydrate Research. 1976;52(1):214-218.

25. Clark JM. Experimental Biochemistry. In: Charles H, et al. (Eds.), North Carolina, USA. 1964.

26. Shepherd MG, Poulter RTM, Sullivan PA. Candida albicans: Biology, Genetics, and Pathogenicity. Annual Review of Microbiology. 1985;39:579-614.

27. Cabib E, Farkas V. The Control of Morphogenesis: An Enzymatic Mechanism for the Initiation of Septum Formation in Yeast. Proc Natl Acad Sci USA. 1971;68(9):2052-2056.

28. Galgiani JN, Claire MP, James FJ. Human PolymorphonuclearLeukocyte Inhibition of Oncorporation of Chitin Precursors Into Mycelia of Coccidilides immitis. The Journal of Infectious Diseases. 1984;149(3):404-412. 
29. Organic Chemistry (A Series of Monographs). Academic Press, New York. 1979.

30. Ward JM. Pespiratory Metabolism of Normal and Divisionless Strains of Candida albicans. Journal of General Physiology. 1958;42:708.

31. Kennedy SI. The Effect of Enzymes on Structural and Biological Properties of Semliki Forest Virus. J Gen Virol. 1974;23(2):129-143.

32. Sasaki T. Convenient Proce $\neg$ dure for the Preparation of Methionine Sulfoxide Derivatives. Peptide Chemistry. 1977. p.15-18.

33. Roy D, Wong PK, Engelbrecht RS, et al. Mechanism of Enteroviral Inactivation by Ozone. Appl Environ Microbiol. 1981;41(3):718-723.

34. Shinriki, N, Kozo I, Akira I, et al. Degradation of Nucleic Acids With Ozone. II. Degradation of Yeast RNA, Yeast Phenylalanine tRN A and Tobacco Mosaic Virus RNA. Biochimica et Biophysica Acta (BBA) Nucleic Acids and Protein Synthesis. 1981;655(2-3):323-328.

35. Koch Y, Rademacher KH. Chemical and Enzymatic Changes in the Cell Walls of Candida albicans and Saccharomyces cerevisiae by Scanning Electron Microscopy. Can J Microbiol. 1980;26(8):965-970.

36. Yamaguchi H. Control of Dimorphism in Candida albicans by Cultures With Differential Biotin Concentrations: Effects of Biotin on Cell Wall Composition and Metabolism. Journal of General Microbiology. $1975 ; 86: 370-372$

37. Hoberg KA, Cihlar RL, Calderone RA. Inhibitory Effect of Cerulenin and Sodium Butyrate on Germination of Candida albicans. Antimicrob Agents Chemother. 1983;24(3):401-408.

38. Kerridge D. The Plasma Membrane of Candida albicans and Its Role in the Action of Anti-Fungal Drugs. Symposium of the Society of General Microbiology. 1980;30:103.
39. Misato. Inhibition of Fungal Cell Wall Synthesis and Cell Membrane Function. Antifungal Compounds. 1977;2:277.

40. Elkhouly AE. Studies on Nystatin Action on Candida albicans Using Scanning Electron Microscope. Mykosen. 1978;21(9):310-324.

41. Shepherd MG, Chin CM, Sullivan PA. The Alternate Respiratory Pathway of Candida albicans. Arch Microbiol. 1978;116:61-67.

42. Odds FC. Morphogenesis in Candida albicans. Crit Rev Microbiol. 1985;12(1):45-93.

43. Rippon JW. Medical Mycology, Saunders, Philadelphia, USA. 1974. p. 177

44. Culbert ML. AIDS: Terror, Truth, Triumph. Chula Vista, California, USA. 1986.

45. Klieneberger NE. Filterable Forms of Bacteria. Bacteriol Rev. 1950;15(2):77-103.

46. Bradford RW. Clinical Management of Epstein-Barr Virus/CFIDS. 1996.

47. Pagano JS. Molecular epidemiology of Epstein-Barr virus infection: A perspective, UCLA Symposium on Molecular and Cellular Biology, New york, USA. 1986;40:345.

48. Carrel A, Dehelly G. The treatment of infected wounds. (2nd edn), 1918, Bailliere, Tindall \& Cox, London, USA. 1918. p.238.

49. Bradford RW. Exogenous Oxidative Mechanisms in Combating Infectious Agents. 1986. 